Article

\title{
Inverse Numerical Range and Determinantal Quartic Curves
}

\author{
Mao-Ting Chien ${ }^{1, *}+\mathbb{( D}$ and Hiroshi Nakazato ${ }^{2,+}$ \\ 1 Department of Mathematics, Soochow University, Taipei 11102, Taiwan \\ 2 Department of Mathematics and Physics, Faculty of Science and Technology, Hirosaki University, \\ Hirosaki 036-8561, Japan; nakahr@hirosaki-u.ac.jp \\ * Correspondence: mtchien@scu.edu.tw \\ + These authors contributed equally to this work.
}

Received: 6 October 2020; Accepted: 24 November 2020; Published: 26 November 2020

check for updates

\begin{abstract}
A hyperbolic ternary form, according to the Helton-Vinnikov theorem, admits a determinantal representation of a linear symmetric matrix pencil. A kernel vector function of the linear symmetric matrix pencil is a solution to the inverse numerical range problem of a matrix. We show that the kernel vector function associated to an irreducible hyperbolic elliptic curve is related to the elliptic group structure of the theta functions used in the Helton-Vinnikov theorem.
\end{abstract}

Keywords: numerical range; kernel vector function; theta function; quartic curve

\section{Introduction}

Let $A$ be an $n \times n$ complex matrix. Toeplitz [1] introduced the numerical range of $A$ as the set

$$
W(A)=\left\{\xi^{*} A \xi: \xi \in \mathbb{C}^{n}, \xi^{*} \xi=1\right\}
$$

which contains all eigenvalues of $A$. The inverse numerical range aims to determine a vector $\xi \in \mathbb{C}^{n}$ satisfying $\xi^{*} A \xi=t_{0} \xi^{*} \xi$ for a moving boundary point $t_{0}$ of $W(A)$. The inverse numerical range problem has been discussed by many authors (see [2-4]). Our approach to this inverse problem is based on the algebraic curve theory and the determinantal representation of a hyperbolic ternary form.

The determinantal ternary form associated to an $n \times n$ matrix $A$ is the homogeneous polynomial $F_{A}(t, x, y)=\operatorname{det}\left(M_{A}(t, x, y)\right)$, where the linear matrix pencil

$$
M_{A}(t, x, y)=t I_{n}+x \Re(A)+y \Im(A),
$$

and $\Re(A)=\left(A+A^{*}\right) / 2, \Im(A)=\left(A-A^{*}\right) /(2 i)$. The algebraic curve of $A$ is the set

$$
\mathcal{V}_{\mathbb{C}}\left(F_{A}\right)=\left\{[(t, x, y)] \in \mathbb{C P}^{2}: F_{A}(t, x, y)=0\right\},
$$

where $[(t, x, y)]$ is the equivalence class of points $(t, x, y) \in \mathbb{C}^{3}$ with $(t, x, y) \neq(0,0,0)$, under the relation that $(t, x, y) \equiv k(t, x, y)$, for $k \in \mathbb{C}, k \neq 0$. The form $F_{A}(t, x, y)$ is hyperbolic with respect to the point $(1,0,0)$, that is, the equation

$$
F_{A}(t,-\cos \theta,-\sin \theta)=\operatorname{det}\left(t I_{n}-\cos \theta \Re(A)-\sin \theta \Im(A)\right)=0
$$

has $n$ real roots counting multiplicities for any $\theta$, and $F(1,0,0)=1$. Note that

$$
F_{A}(t,-\cos \theta,-\sin \theta)=\operatorname{det}\left(t I_{n}-\Re\left(e^{-i \theta} A\right)\right) \text {, }
$$


the characteristic polynomial of the Hermitian matrix $\Re\left(e^{-i \theta} A\right)$.

Kippenhahn [5] proved that the numerical range $W(A)$ is the convex hull of the real affine part of the dual curve of $F_{A}(t, x, y)=0$. Conversely, Lax [6] conjectured that every hyperbolic ternary form $F(t, x, y)$ of degree $n$ admits a determinantal representation by a linear matrix pencil $M(t, x, y)=t I_{n}+x C+y B$ of real symmetric matrices $C$ and $B$, i.e.,

$$
F(t, x, y)=\operatorname{det}\left(t I_{n}+x C+y B\right) .
$$

Fiedler [7] proved that the Lax conjecture is true if $\mathcal{V}_{\mathbb{C}}(F)$ is a rational curve, and raised a similar conjecture in a relaxed form where $B$ and $C$ are Hermitian matrices. Plaumann and Vinzant [8] provided a method to construct a linear matrix pencil with Hermitian matrices $B, C$ using the interlacer $\frac{\partial}{\partial t} F(t, x, y)$ of the hyperbolic form $F(t, x, y)$. Recently, Helton and Vinnikov [9] confirmed that the Lax conjecture is true by algebraic curve theory for the construction of real symmetric matrices $B$ and $C$ using Riemann-Jacobi theta functions with characteristics (see also $[10,11]$ ).

It is well known that any boundary point $t_{0}$ of $W(A)$ corresponds to an extreme eigenvalue of $\Re\left(e^{-i \theta} A\right)$ for some angle $\theta$. A unital eigenvector $\xi_{\theta}$ of $\Re\left(e^{-i \theta} A\right)$ corresponding to the maximal eigenvalue $\lambda_{\max }\left(\Re\left(e^{-i \theta} A\right)\right)$ assures $\xi_{\theta}^{*} A \xi_{\theta} \in \partial W(A)$, and

$$
M_{A}\left(\lambda_{\max }\left(\Re\left(e^{-i \theta} A\right)\right),-\cos \theta,-\sin \theta\right) \xi_{\theta}=0 .
$$

From this view point, the inverse numerical range problem can be renamed in a more general setting: For any nonzero point $(t, x, y) \in \mathbb{C}^{3}$ on the curve $F_{A}(t, x, y)=0$, to find a kernel vector function $\xi \in \mathbb{C}^{n}$ satisfying

$$
M_{A}(t, x, y) \xi=\left(t I_{n}+x \Re(A)+y \Im(A)\right) \xi=0 .
$$

The kernel vector function method is used in [12] to deal with the inverse numerical range of a $3 \times 3$ matrix for which its algebraic curve is a cubic elliptic curve.

In this paper, we continue our work on the inverse numerical range problem using the kernel vector function method in the case that $\mathcal{V}_{\mathbb{C}}\left(F_{A}\right)$ is a quartic elliptic curve. We show that the intersection points of the kernel vector functions and the algebraic curve $\mathcal{V}_{\mathbb{C}}\left(F_{A}\right)$ induce an abelian group structure on the Abel-Jacobi variety, and the kernel vector functions can be expressed in terms of the theta functions used in the Helton-Vinnikov theorem.

\section{Quartic Elliptic Curves}

Let $F(t, x, y)$ be an irreducible hyperbolic form of degree $n$. A point $\left(t_{0}, x_{0}, y_{0}\right)$ is called a singular point of the curve $\mathcal{V}_{\mathbb{C}}(F)$ if

$$
F\left(t_{0}, x_{0}, y_{0}\right)=\frac{\partial}{\partial t} F\left(t_{0}, x_{0}, y_{0}\right)=\frac{\partial}{\partial x} F\left(t_{0}, x_{0}, y_{0}\right)=\frac{\partial}{\partial y} F\left(t_{0}, x_{0}, y_{0}\right)=0 .
$$

A singular point $\left(t_{0}, x_{0}, y_{0}\right)$ is called an ordinary double point if the Hessian matrix $H_{F}\left(t_{0}, x_{0}, y_{0}\right)$ of $F(t, x, y)$ at $\left(t_{0}, x_{0}, y_{0}\right)$ is invertible. The curve $\mathcal{V}_{\mathbb{C}}(F)$ is elliptic if it has genus 1 . (For reference on algebraic curve theory, see, for instance, [13].)

We recall some previous results on the determinantal representation of elliptic curves in [11]. Assume that the curve $\mathcal{V}_{\mathbb{C}}(F)$ is elliptic, and the $n$ real intersection points of the curve $F(t, x, y)=0$ and the line $x=0$ are distinct non-singular points $Q_{1}, \ldots, Q_{n}$ with coordinates $Q_{j}=\left(b_{j}, 0,-1\right)$, where $b_{j} \neq 0$. Then there is a real birational transformation which transforms $\mathcal{V}_{\mathbb{C}}(F)$ to a non-singular cubic curve $Y^{2} Z=4 X^{3}-g_{2} X^{2} Z-g_{3} Z^{3}$ for some real constants $g_{2}, g_{3}$ with $g_{2}^{3}-27 g_{3}^{2}>0$. The real affine part $F(1, x, y)=0$ of the curve $\mathcal{V}_{\mathbb{C}}(F)$ is then parametrized by rational functions of Weierstrass function and its derivative over the torus. The Abel-Jacobi map $\phi: \mathcal{V}_{\mathbb{C}}(F) \rightarrow \operatorname{Jac}(X)$ is the inverse of the parametrization $s \mapsto(1, x, y)$. Denote $Q_{j}^{\prime}=\phi\left(Q_{j}\right)$. 
In the paper [11], the Helton-Vinnikov representation for an elliptic curve is formulated by using the Riemann-Jacobi theta functions $\theta_{\delta}(v)$ on the normalized Abel-Jacobi variety $\mathbb{C} /(\mathbb{Z}+\tau \mathbb{Z}), \tau \in i \mathbb{R}$, $\Im(\tau)>0$. The theta function $\theta_{1}$ is defined by

$$
\theta_{1}(v)=2 q^{1 / 4} \sum_{m=0}^{\infty}(-1)^{m} q^{(m+1) m} \sin ((2 m+1) \pi v)
$$

where $q=\exp (i \pi \tau)$. The relations among $\theta_{\delta}(v)$ functions are related as follows.

$$
\begin{aligned}
& \theta_{2}(v)=\theta_{1}\left(v+\frac{1}{2}\right) \\
& \theta_{3}(v)=i q^{-1 / 4} \exp (-i \pi v) \theta_{1}\left(v-\frac{1}{2}-\frac{\tau}{2}\right) \\
& \theta_{4}(v)=i q^{-1 / 4} \exp (-i \pi v) \theta_{1}(v-\tau / 2),
\end{aligned}
$$

$0 \leq \Re(v) \leq 1,0 \leq \Im(v) \leq \Im(\tau)$. The main result of [11] reformulates the Helton-Vinnikov determinantal representation as follows.

Theorem 1 ([11], Theorem 2.4). Let $F(t, x, y)$ be an irreducible hyperbolic ternary form of degree $n$. Assume that the curve $\mathcal{V}_{\mathbb{C}}(F)$ intersects the line $x=0$ at $n$ distinct nonsingular points $Q_{j}=\left(b_{j}, 0,-1\right)$ with $b_{j} \neq 0, j=1,2, \ldots, n$, and assume that $\mathcal{V}_{\mathbb{C}}(F)$ is an elliptic curve which is parametrized as $x=R_{1}\left(\wp(u), \wp^{\prime}(u)\right), y=R_{2}\left(\wp(u), \wp^{\prime}(u)\right)$ by two rational functions $R_{1}, R_{2}$. Let $Q_{j}^{\prime}=\phi\left(Q_{j}\right)$ be the point of the torus $\mathbb{T}$ corresponding to the point $Q_{j}$, and $B=\operatorname{diag}\left(b_{1}, b_{2}, \ldots, b_{n}\right)$. For $\delta=2,3$, the off-diagonal entries of the matrix $C=\left(c_{i j}\right)$ in the determinantal representation (1) are given by

$$
\begin{gathered}
c_{j k}=\frac{\left(b_{k}-b_{j}\right) \theta_{1}^{\prime}(0)}{2 \omega_{1} \theta_{\delta}(0)} \cdot \frac{\theta_{\delta}\left(\left(Q_{k}^{\prime}-Q_{j}^{\prime}\right) /\left(2 \omega_{1}\right)\right)}{\theta_{1}\left(\left(Q_{k}^{\prime}-Q_{j}^{\prime}\right) /\left(2 \omega_{1}\right)\right)} \cdot \frac{1}{\sqrt{d\left(\frac{R_{1}}{R_{2}}\right)\left(Q_{j}^{\prime}\right)} \sqrt{d\left(\frac{R_{1}}{R_{2}}\right)\left(Q_{k}^{\prime}\right)}}, j \neq k, \\
c_{j j}=b_{j} \frac{F_{x}\left(b_{j}, 0,-1\right)}{F_{y}\left(b_{j}, 0,-1\right)},
\end{gathered}
$$

where $\omega_{1}>0$ is a half-period of the Weierstrass function $\wp(u)$.

We raise the following conjecture that the kernel vector functions play a role for inverse numerical range and formulate the determinantal representation as well.

Let Conjecture 1. $A$ be an $n \times n$ matrix. Assume that the skew Hermitian matrix $\Im(A)$ is diagonal with distinct diagonals $b_{1}, b_{2}, \ldots, b_{n}$. We also assume that the ternary form $F_{A}(t, x, y)$ is irreducible and its algebraic curve $\mathcal{V}_{\mathbb{C}}\left(F_{A}\right)$ is elliptic. Then there exist $n$ points $R_{1}^{\prime}, R_{2}^{\prime}, \ldots, R_{n}^{\prime}$ in the normalized Abel-Jacobi variety $\mathbb{C} /(\mathbb{Z}+\tau \mathbb{Z})$ of the elliptic curve for which the reduced kernel vector function $\left(\tilde{\xi}_{1}, \tilde{\xi}_{2}, \ldots, \tilde{\xi}_{n}\right)^{T}$ is expressed as

$$
\tilde{\xi}_{k}=C_{k}\left(\prod_{1 \leq j \leq n, j \neq k} \theta_{1}\left(s-Q_{j}^{\prime}\right)\right) \theta_{1}\left(s-R_{k}^{\prime}\right),
$$

for some constants $C_{k} \neq 0, k=1,2, \ldots, n$, on the Abel-Jacobi variety. With respect to the abelian group structure of this variety, one has

$$
R_{1}^{\prime}-Q_{1}^{\prime} \equiv R_{2}^{\prime}-Q_{2}^{\prime} \equiv \cdots \equiv R_{n}^{\prime}-Q_{n}^{\prime}
$$

If the linear matrix pencil $M(t, x, y)$ is unitarily equivalent to the matrix pencil realized as the $\theta_{\delta}$-Helton Vinikov representation for $\delta=2$ or $\delta=3$, then $\left(R_{1}^{\prime}-Q_{1}^{\prime}\right)+\left(R_{1}^{\prime}-Q_{1}^{\prime}\right)=0$. Furthermore, the point $R_{1}^{\prime}-Q_{1}^{\prime}$ coincides with the point $1 / 2$ (resp. $(1+\tau) / 2$ ) of the normalized Abel-Jacobi variety when $\delta=2$ (resp. $\delta=3)$. 
The result of a previous paper [12] shows that the conjecture is true for cubic elliptic curves. In this section, we confirm that the conjecture is also true for some quartic elliptic curves.

In the paper [14], a $4 \times 4$ nilpotent matrix

$$
\left(\begin{array}{cccc}
0 & 2 \sqrt{s} /(1-s) & 0 & 2 /(1-s) \\
0 & 0 & 2 /(1-s) & 0 \\
0 & 0 & 0 & 2 \sqrt{s} /(1-s) \\
0 & 0 & 0 & 0
\end{array}\right)
$$

$0<s<1$, is studied which produces one type of numerical ranges for the classification of the numerical ranges of $4 \times 4$ matrices. Fladt [15] formulated this quartic curve $F_{A}(1, x, y)=0$ as one of the Kepler's models of planetary orbits. We consider a more general similar form of the matrix (2a):

$$
A=\left(\begin{array}{cccc}
a & a_{12} & a_{13} & a_{14} \\
0 & -a & -a_{14} & a_{13} \\
0 & 0 & i a & -a_{12} \\
0 & 0 & 0 & -i a
\end{array}\right)
$$

where $a>0$ and $a_{12}, a_{13}, a_{14}$ are real numbers.

Theorem 2. Let $A$ be the matrix defined in (2b). If $a_{13}=a_{12} a_{14} /(2 a)$ then the algebraic curve $F_{A}(1, x, y)=0$ has a pair of ordinary double points on the line $y=x$. In this case, the two ordinary double points are $(1, w, w)$ and $(1,-w,-w)$, where $w=\frac{2 \sqrt{2} a}{\left(a_{12}^{2} a_{14}^{2}+4 a^{2}\left(a_{12}^{2}+a_{14}^{2}\right)+8 a^{4}\right)^{1 / 2}}$.

Proof. Assume $(1, w, w)$ is an ordinary double point of $F_{A}(1, x, y)=0$. Then the resultant of $F_{A}(t, x, y)$ and $\frac{\partial}{\partial x} F_{A}(t, x, y)$ with respect to $x$ for $x=y$ is 0 . Using "Resultant" function of the "Mathematica" software, the resultant is given by

$$
\begin{gathered}
16777216\left(2 a a_{13}-a_{12} a_{14}\right)^{4}\left(8 a^{4}+8 a^{2} a_{12}^{2}+12 a_{12}^{4}-8 a^{2} a_{13}^{2}+4 a_{12}^{2} a_{13}^{2}+2 a_{13}^{4}\right. \\
\left.+24 a a_{12} a_{13} a_{24}+8 a^{2} a_{14}^{2}-5 a_{12}^{2} a_{14}^{2}+4 a_{13}^{2} a_{14}^{2}+2 a_{14}^{4}\right)^{2} t^{12}
\end{gathered}
$$

Clearly, the condition $a_{13}=a_{12} a_{14} /(2 a)$ assures that the resultant is 0 .

One easy way to find an ordinary double point $(1, w, w)$ is computing the zeros of $\frac{\partial}{\partial t} F_{A}(t, x, y)$ at $(1, w, w)$. One factor of $\frac{\partial}{\partial t} F_{A}(t, x, y)$ for $t=1$ and $x=y=w$ is given by

$$
8 a^{2}-8 a^{4} w^{2}-4 a^{2} a_{12}^{2} w^{2}-4 a^{2} a_{14}^{2} w^{2}-a_{12}^{2} a_{14}^{2} w^{2} .
$$

Its zeros are $w= \pm \frac{2 \sqrt{2} a}{\left(a_{12}^{2} a_{14}^{2}+4 a^{2}\left(a_{12}^{2}+a_{14}^{2}\right)+8 a^{4}\right)^{1 / 2}}$, and the points $(1, w, w)$ are also roots of $F_{A}(t, x, y)=\frac{\partial}{\partial x} F_{A}(t, x, y)=\frac{\partial}{\partial y} F_{A}(t, x, y)=0$.

For simplicity, we present a numerical computation which confirms Conjecture 1. This computation method can be used for employing general computation for a generic quartic curve with two ordinary double points. For this purpose, we define the $4 \times 4$ matrix $A$ which is the form of ( $2 b)$ satisfying the assumption $a_{13}=a_{12} a_{14} /(2 a)$ of Theorem 2 with special values $a=(2 \sqrt{2} / 3 \times 17)^{1 / 4}, a_{12}=\left(\frac{20 \sqrt{17}-66}{17}\right)^{1 / 2}$ and $a_{14}=8\left(\frac{10 \sqrt{17}-1}{1699}\right)^{1 / 2}$. Then

$$
\begin{gathered}
81 F_{2}(t, x, y) \equiv 81 F_{A}(t, x, y) \\
=81 t^{4}-720 t^{2}\left(x^{2}+y^{2}\right)+816\left(x^{4}+y^{4}\right)+2720 x^{2} y^{2}+1024 x y\left(x^{2}+y^{2}\right) .
\end{gathered}
$$


The corresponding curve has just two singular points (ordinary double points) lying on the line $y=x$. According to the genus formula [13] of an algebraic curve:

$$
g\left(\mathcal{V}_{\mathbb{C}}\left(F_{2}\right)\right)=\frac{1}{2}(n-1)(n-2)-\text { number of ordinary double points }=1,
$$

it implies that the quartic curve $\mathcal{V}_{\mathbb{C}}\left(F_{2}\right)$ is an elliptic curve.

In the following, we show that the quartic form $F_{2}(t, x, y)$ admits two non-unitarily equivalent determinantal representations.

Theorem 3. Let $F_{2}(t, x, y)$ be the quartic form in (2c). Then, there exist two real symmetric matrices $H_{1}$ and $H_{2}$, such that $H_{1}+i B$ and $H_{2}+i B$ are not unitarily equivalent, and $F_{2}(t, x, y)=\operatorname{det}\left(t I_{4}+x H_{j}+y B\right)$, $j=1,2$, where $B=\operatorname{diag}(2 \sqrt{17} / 3,-2 \sqrt{17} / 3,2 / \sqrt{3},-2 / \sqrt{3})$, and

$$
H=\left(\begin{array}{cccc}
h_{11} & h_{12} & h_{13} & h_{14} \\
h_{12} & -h_{11} & h_{14} & -h_{13} \\
h_{13} & h_{14} & h_{33} & h_{34} \\
h_{14} & -h_{13} & h_{34} & -h_{33}
\end{array}\right)
$$

with entries $h_{11}=32 /(21 \sqrt{17}), h_{33}=32 /(21 \sqrt{3})$ and the quartet $\left(h_{12}, h_{13}, h_{14}, h_{34}\right)$ according to the two inequivalent representations: One matrix $H=H_{1}$ is given by

$$
\left(h_{12}, h_{13}, h_{14}, h_{34}\right)=\left(\frac{2}{21} \sqrt{\frac{611}{17}}, \frac{8}{21} \sqrt{\frac{611}{510+67 \sqrt{51}}}, \frac{8}{21} \sqrt{\frac{510+67 \sqrt{51}}{51}},-\frac{2}{21} \sqrt{\frac{611}{3}}\right),
$$

and another matrix $\mathrm{H}=\mathrm{H}_{2}$ is given by

$$
\left(h_{12}, h_{13}, h_{14}, h_{34}\right)=\left(-\frac{6}{7} \sqrt{\frac{47}{17}}, \frac{2}{7} \sqrt{\frac{1034}{3(51+2 \sqrt{51})}}, \frac{2}{21} \sqrt{\frac{22(51+2 \sqrt{51})}{17}},-\frac{10}{21} \sqrt{\frac{47}{3}}\right) .
$$

Proof. By comparing the traces,

$$
\begin{aligned}
& \operatorname{trace}\left(\left(\left(H_{1}+i B\right)\left(H_{1}-i B\right)\right)^{3}\right)=7147520 / 729, \\
& \operatorname{trace}\left(\left(\left(H_{2}+i B\right)\left(H_{2}-i B\right)\right)^{3}\right)=8302592 / 729,
\end{aligned}
$$

the unequal traces ascertain that $H_{1}+i B$ and $H_{2}+i B$ are non-unitarily equivalent. The diagonal matrix $B$ whose diagonal entries are the roots of $F_{2}(t, 0,-1)=0$, i.e.,

$$
81 t^{4}-720 t^{2}+816=0
$$

which gives $t= \pm 2 \sqrt{17} / 3, \pm 2 / \sqrt{3}$.

Next, we determine the symmetric matrix $H=\left(h_{i j}\right)$ satisfying $h_{23}=h_{14}$ and $h_{24}=-h_{13}$. For this, we compute

$$
\begin{gathered}
81 \operatorname{det}\left(t I_{4}+x H+y B\right)-81 F_{2}(t, x, y) \\
=\frac{x^{2}}{122451}\left[441 C_{1} t^{2}+196 C_{2} y^{2}+392 C_{3} x y+C_{4} x^{2}\right],
\end{gathered}
$$

where the polynomials $C_{j}=C_{j}\left(h_{12}, h_{13}, h_{14}, h_{34}\right)$ are given by the following: 


$$
\begin{aligned}
C_{1}= & 179440-22491\left(h_{12}^{2}+h_{34}^{2}\right)-44982\left(h_{13}^{2}+h_{14}^{2}\right) \\
C_{2}= & -1603064+67473 h_{12}^{2}+44982 \sqrt{51}\left(h_{14}^{2}-h_{13}^{2}\right)+382347 h_{34}^{2} \\
C_{3}= & -352640+51408\left(h_{12}^{2}-h_{34}^{2}\right)-67473\left(\sqrt{3} h_{12} h_{13} h_{14}+\sqrt{17} h_{13} h_{14} h_{34}\right) \\
& +14112 \sqrt{51}\left(h_{14}^{2}-h_{13}^{2}\right), \\
C_{4}= & -98871440+7676928 h_{12}^{2}+903168 \sqrt{51}\left(h_{13}^{2}-h_{14}^{2}\right)-20151936 \sqrt{3} h_{12} h_{13} \\
& +19837062\left(h_{13}^{2} h_{14}^{2}+h_{12} h_{13}^{2} h_{34}-h_{12} h_{14}^{2} h_{34}\right)+1354752 h_{34}^{2} \\
& +9918531\left(h_{13}^{4}+h_{14}^{2}+h_{12}^{2} h_{34}^{2}\right)+3556224 \sqrt{17} h_{13} h_{14} h_{34} .
\end{aligned}
$$

To prove $F_{2}(t, x, y)=\operatorname{det}\left(t_{4}+x H_{j}+y B\right)$, it suffices to show that the two real quartets $\left(h_{12}, h_{13}, h_{14}, h_{34}\right)$ satisfy the simultaneous equations $C_{1}=C_{2}=C_{3}=C_{4}=0$. The computations of the Groebner basis of the ideal $\left(C_{1}, C_{2}, C_{3}, C_{4}\right)$ of the polynomial ring $\mathbb{C}\left[h_{12}, h_{13}, h_{14}, h_{34}\right]$ is efficient to solve the system, and direct computations show that the two quartets are solutions of the simultaneous equations.

We present a numerical computation for the quartic form $F_{2}(t, x, y)$ which confirms the Conjecture 1.

Theorem 4. Let $B$ and $\mathrm{H}_{2}$ be matrices defined in Theorem 3, and let the linear matrix pencil $M(t, x, y)=$ $t I_{4}+x H_{2}+y B$. Assume $\xi=\left(\xi_{1}, \xi_{2}, \xi_{3}, \xi_{4}\right)^{T}$ is the 4 -th row of the adjugate matrix of $M(t, x, y)$. Then there are 10 distinct intersecting real points $Q_{1}, Q_{2}, Q_{3}, Q_{4}, R_{1}, R_{2}, R_{3}, R_{4}, S_{1}, S_{2}$ on the curves $F_{2}(t, x, y)(=$ $\operatorname{det}(M(t, x, y)))=0$ and $\xi_{j}(t, x, y)=0$. Furthermore, their corresponding points on the normalized Abel-Jacobi variety of the quartic curve $\operatorname{det}(M(t, x, y)=0$ satisfy the elliptic curve theoretic property:

$$
Q_{1}^{\prime}+Q_{2}^{\prime} \equiv Q_{3}^{\prime}+Q_{4}^{\prime} \equiv R_{1}^{\prime}+R_{2}^{\prime} \equiv R_{3}^{\prime}+R_{4}^{\prime} \equiv E_{2},
$$

and

$$
R_{1}^{\prime}-Q_{1}^{\prime} \equiv R_{2}^{\prime}-Q_{2}^{\prime} \equiv R_{3}^{\prime}-Q_{3}^{\prime} \equiv R_{4}^{\prime}-Q_{4}^{\prime} \equiv E_{1},
$$

where $E_{2} \equiv(1+\tau) / 2$ and $E_{1} \equiv 1 / 2$ on the normalized Abel-Jacobi variety $\mathbb{C} /(\mathbb{Z}+\tau \mathbb{Z})$.

Proof. We construct a kernel vector function $\xi=\left(\xi_{1}, \xi_{2}, \xi_{3}, \xi_{4}\right)$ by computing the 4 -th row of the adjugate matrix of $M(t, x, y)$, which is given by

$$
\begin{aligned}
\xi_{1}= & -\frac{2}{189} \sqrt{\frac{22}{51+2 \sqrt{51}}} x\left((-24 \sqrt{17}+172 \sqrt{3}) x^{2}+(-192 \sqrt{17}-128 \sqrt{3}) x y\right. \\
& \left.+(-6 \sqrt{51}+270) x t-(24 \sqrt{17}+204 \sqrt{3}) y^{2}+(6 \sqrt{51}-270) y t+(27 \sqrt{17}+18 \sqrt{3}) t^{2}\right), \\
\xi_{2}= & -\frac{2 \sqrt{1034}}{63}(51+2 \sqrt{51})^{3 / 2} x\left((236 \sqrt{17}+408 \sqrt{3}) x^{2}+(128 \sqrt{17}+1088 \sqrt{3}) x y\right. \\
& +(114 \sqrt{51}+510 x z t)+(-204 \sqrt{17}-136 \sqrt{3}) y^{2}-(114 \sqrt{51}+510) y t \\
& \left.-(18 \sqrt{17}+153 \sqrt{3}) t^{2}\right), \\
\xi_{3}= & -\frac{2 \sqrt{47}(17 \sqrt{3}+2 \sqrt{17})}{189(51+2 \sqrt{51})} x\left(76 x^{2}-16 x y+340 y^{2}-45 t^{2}\right), \\
\xi_{4}= & -\frac{1}{189(51+2 \sqrt{51})}\left((2240 \sqrt{17}+19040 \sqrt{3}) x^{3}+(1072 \sqrt{17}+9112 \sqrt{3}) x^{2} y\right. \\
& +(1272 \sqrt{51}+32436) x^{2} t+(2816 \sqrt{17}+23936 \sqrt{3}) x y^{2}-(768 \sqrt{51}+19584) x y t \\
& -(576 \sqrt{17}+4896 \sqrt{3}) x t^{2}+(5712 \sqrt{17}+48552 \sqrt{3}) y^{3}+(2856 \sqrt{51}+72828) y^{2} t \\
& \left.-(756 \sqrt{17}+6426 \sqrt{3}) y t^{2}-(378 \sqrt{51}+9639) t^{3}\right) .
\end{aligned}
$$


The intersection points including multiplicities of the curve $F_{2}(t, x, y)=0$ and the curves $\xi_{j}(t, x, y)=0$ are represented by the following divisors:

$$
\begin{aligned}
& F_{2} \cdot \xi_{1}=Q_{1}+2 Q_{2}+2 Q_{3}+Q_{4}+R_{1}+R_{4}+2 S_{1}+2 S_{2} \\
& F_{2} \cdot \xi_{2}=2 Q_{1}+Q_{2}+2 Q_{3}+Q_{4}+R_{2}+R_{4}+2 S_{1}+2 S_{2} \\
& F_{2} \cdot \xi_{3}=2 Q_{1}+2 Q_{2}+Q_{4}+R_{3}+R_{4}+2 S_{1}+2 S_{2} \\
& F_{2} \cdot \xi_{4}=2 Q_{1}+2 Q_{2}+2 Q_{3}+2 R_{4}+2 S_{1}+2 S_{2}
\end{aligned}
$$

where the 10 points $Q_{1}, Q_{2}, Q_{3}, Q_{4}, R_{1}, R_{2}, R_{3}, R_{4}, S_{1}, S_{2}$ are mutually distinct real points of the curve $F_{2}(t, x, y)=0$ whose $(t, x, y)$-coordinates are given by

$$
\begin{gathered}
Q_{1}=\left(1,0,-\frac{3}{2 \sqrt{17}}\right), Q_{2}=\left(1,0, \frac{3}{2 \sqrt{17}}\right), Q_{3}=\left(1,0,-\frac{\sqrt{3}}{2}\right), Q_{4}=\left(1,0, \frac{\sqrt{3}}{2}\right), \\
R_{1}=\left(1,-\frac{9}{4 \sqrt{17}}, \frac{15}{4 \sqrt{17}}\right), R_{2}=\left(1, \frac{9}{4 \sqrt{17}},-\frac{15}{4 \sqrt{17}}\right), R_{3}=\left(1, \frac{5}{4 \sqrt{3}}, \frac{1}{4 \sqrt{3}}\right), \\
R_{4}=\left(1,-\frac{5}{4 \sqrt{3}},-\frac{1}{4 \sqrt{3}}\right), S_{1}=\left(1, \frac{3}{4 \sqrt{5}}, \frac{3}{4 \sqrt{5}}\right), S_{2}=\left(1,-\frac{3}{4 \sqrt{5}},-\frac{3}{4 \sqrt{5}}\right) .
\end{gathered}
$$

Hence, the reduced divisors $\tilde{\xi}_{j}$ of $F_{2} \cdot \xi_{j}$ by removing the common multiplicities of the zero points are given by

$$
\begin{aligned}
& \tilde{\xi}_{1}=Q_{2}+Q_{3}+Q_{4}+R_{1}, \\
& \tilde{\xi}_{2}=Q_{1}+Q_{3}+Q_{4}+R_{2}, \\
& \tilde{\xi}_{3}=Q_{1}+Q_{2}+Q_{4}+R_{3}, \\
& \tilde{\xi}_{4}=Q_{1}+Q_{2}+Q_{3}+R_{4} .
\end{aligned}
$$

Note that the numerical intersection points of the curve $F_{2}(t, x, y)=0$ and the curve $\xi_{j}(t, x, y)=0$ can be achieved by applying NSolve function of Mathematica. For instance, let $t=1$, NSolve $\left[F_{2}==\right.$ $\left.0, \xi_{j}==0,\{x, y\}\right]$ produces numerical solutions. The performance of symbolic computations of the intersection points needs more delicate treatments. Using the Resultant function, we can get the algebraic equations defining the coordinates of the intersection point $(1, x, y)$.

We are now ready to prove that the points $Q_{j}^{\prime}$ and $R_{k}^{\prime}$ on the normalized Abel-Jacobi variety of the quartic curve $F_{2}(t, x, y)=0$ correspond to $Q_{j}$ and $R_{k}$ satisfy the elliptic curve theoretic property (2d) and (2e).

Firstly, we perform successive real projective and real Cremona transformations. The ternary form

$$
81 F_{2}(t, x, y)=81 t^{4}-720\left(x^{2}+y^{2}\right) t^{2}+816 x^{4}+1024 x y\left(x^{2}+y^{2}\right)+2720 x^{2} y^{2}+816 y^{4}
$$

is transformed to

$$
81 K_{0}(T, X, Y)=81 T^{4}-360 T^{2}\left(X^{2}+Y^{2}\right)+144 X^{4}+272 X^{2} Y^{2}+400 Y^{4}
$$

under changing the variables $T=t, X=-x+y, Y=x+y$. The points $Q_{j}$ and $R_{k}$ are transformed on the line $X=Y$ in $(T, X, Y)$-coordinates.

$$
Q_{1}=\left(1,-\frac{3}{2 \sqrt{17}},-\frac{3}{2 \sqrt{17}}\right), Q_{2}=\left(1, \frac{3}{2 \sqrt{17}}, \frac{3}{2 \sqrt{17}}\right), Q_{3}=\left(1,-\frac{\sqrt{3}}{2},-\frac{\sqrt{3}}{2}\right), Q_{4}=\left(1, \frac{\sqrt{3}}{2}, \frac{\sqrt{3}}{2}\right) .
$$

The points $R_{j}$ in $(T, X, Y)$-coordinates are given by

$$
R_{1}=\left(1, \frac{6}{\sqrt{17}}, \frac{3}{2 \sqrt{17}}\right), R_{2}=\left(1,-\frac{6}{\sqrt{17}},-\frac{3}{2 \sqrt{17}}\right), R_{3}=\left(1,-\frac{1}{\sqrt{3}}, \frac{\sqrt{3}}{2}\right), R_{4}=\left(1, \frac{1}{\sqrt{3}},-\frac{\sqrt{3}}{2}\right) .
$$

Then, changing the variables

$$
\phi=-2 X, \xi=X+\frac{T}{2}+\frac{\sqrt{5}}{3} Y, \eta=X+\frac{T}{2}-\frac{\sqrt{5}}{3} Y,
$$


the quartic form $K_{0}(T, X, Y)$ is expressed as

$$
\begin{aligned}
\tilde{K}(\phi, \xi, \eta)= & 80 \phi^{3}(\xi+\eta)+147 \phi^{2}\left(\xi^{2}+\eta^{2}\right)+586 \phi^{2} \xi \eta \\
& +720\left(\phi \xi^{2} \eta+\phi \xi \eta^{2}+\xi^{2} \eta^{2}\right) .
\end{aligned}
$$

The corresponding 8 points of $Q_{j}$ and $R_{k}$ on the quartic curve $\tilde{K}(\phi, \xi, \eta)=0$ are respectively expressed as:

and

$$
\begin{gathered}
\left(1, \frac{1}{6}(-3-\sqrt{5}+\sqrt{17}), \frac{1}{6}(-3+\sqrt{5}+\sqrt{17})\right),\left(1, \frac{1}{6}(-3-\sqrt{5}-\sqrt{17}), \frac{1}{6}(-3+\sqrt{5}-\sqrt{17})\right) \\
\left(1, \frac{1}{6}(-3+\sqrt{3}-\sqrt{5}), \frac{1}{6}(-3+\sqrt{3}+\sqrt{5})\right),\left(1, \frac{1}{6}(-3-\sqrt{3}-\sqrt{5}), \frac{1}{6}(-3-\sqrt{3}+\sqrt{5})\right)
\end{gathered}
$$

$$
\begin{gathered}
\left(1, \frac{1}{24}(-12-\sqrt{5}-\sqrt{17}), \frac{1}{24}(-12+\sqrt{5}-\sqrt{17})\right),\left(1, \frac{1}{24}(-12-\sqrt{5}+\sqrt{17}), \frac{1}{24}(-12+\sqrt{5}+\sqrt{17})\right), \\
\left(1, \frac{1}{4}(-2+\sqrt{3}+\sqrt{5}), \frac{1}{4}(-2+\sqrt{3}-\sqrt{5})\right),\left(1, \frac{1}{4}(-2-\sqrt{3}+\sqrt{5}), \frac{1}{4}(-2-\sqrt{3}-\sqrt{5})\right) .
\end{gathered}
$$

Finally, using the Cremona transformation:

$$
u=\xi \eta, \quad v=\phi \eta, \quad w=\phi \xi
$$

and its inverse transformation:

$$
\phi=v w, \quad \xi=u w, \quad \eta=u v,
$$

the quartic curve $\tilde{K}(\phi, \xi, \eta)=0$ is transformed into a non-singular cubic curve

$$
F_{3}(u, v, w)=720\left(u^{3}+u^{2} v+u^{2} w\right)+147\left(u v^{2}+u w^{2}\right)+586 u v w+80\left(v^{2} y+x w^{2}\right)=0
$$

The 8 points $Q_{j}, R_{k}$ on $\tilde{K}(\phi, \xi, \eta)=0$ are transformed to $Q_{j}^{\prime}, R_{k}^{\prime}$ on the cubic curve $F_{3}(u, v, w)=0$ according to the Cremona transformation which are given in $(u, v, w)$-coordinates:

$$
\begin{gathered}
Q_{1}^{\prime}=\left(1, \frac{-6}{3+\sqrt{5}-\sqrt{17}}, \frac{6}{-3+\sqrt{5}+\sqrt{17}}\right), Q_{2}^{\prime}=\left(1, \frac{-6}{3+\sqrt{5}+\sqrt{17}}, \frac{6}{-3+\sqrt{5}-\sqrt{17}}\right), \\
Q_{3}^{\prime}=\left(1, \frac{6}{-3+\sqrt{3}-\sqrt{5}}, \frac{6}{-3+\sqrt{3}+\sqrt{5}}\right), Q_{4}^{\prime}=\left(1, \frac{-6}{3+\sqrt{3}+\sqrt{5}}, \frac{-6}{3+\sqrt{3}-\sqrt{5}},\right. \\
R_{1}^{\prime}=\left(1, \frac{-24}{12+\sqrt{5}+\sqrt{17}}, \frac{24}{-12+\sqrt{5}-\sqrt{17}}\right), R_{2}^{\prime}=\left(1, \frac{-24}{12+\sqrt{5}-\sqrt{17}}, \frac{24}{-12+\sqrt{5}+\sqrt{17}}\right), \\
R_{3}^{\prime}=\left(1, \frac{4}{-2+\sqrt{3}+\sqrt{5}}, \frac{4}{-2+\sqrt{3}-\sqrt{5}}\right), R_{4}^{\prime}=\left(1, \frac{-4}{2+\sqrt{3}-\sqrt{5}}, \frac{-4}{2+\sqrt{3}+\sqrt{5}}\right) .
\end{gathered}
$$

The cubic curve $F_{3}(u, v, w)=0$ has a point of reflection at $(u, v, w)=(0,1,-1)$ which is the neutral element of the elliptic curve group. On the line $v=w$, the cubic curve has 3 points $E_{j}$ which satisfy $E_{j}+E_{j}=0, j=1,2,3$, with respect to the elliptic curve group structure. Among them, $E_{1}=$ $\left(1,-\frac{3}{2},-\frac{3}{2}\right)$ lies on the pseudo-line part of the real cubic curve $\mathcal{V}_{\mathbb{R}}\left(F_{3}\right), E_{2}:\left(t_{0}, x_{0}, y_{0}\right)=(1,-1,-1)$ lies on the oval part of the real cubic curve corresponding to the point $(1+\tau) / 2$ on the normalized Abel-Jacobi variety, and $E_{3}=(1,-3,-3)$ corresponds to $\tau / 2$ on the Abel-Jacobi variety.

To prove (2e) for $E_{1}$, we shall determine the line $K_{j}: a_{j} v+b_{j} w+c_{j} u=0$ passing through $R_{j}^{\prime}$ and $-Q_{j}^{\prime}$ on the cubic curve $F_{3}(u, v, w)=0$. It suffices to show that

$$
-\frac{3}{2} a_{j}-\frac{3}{2} b_{j}+c_{j}=0,
$$

$j=1,2,3,4$. In fact, the lines $K_{j}$ are given by

$$
\begin{aligned}
& K_{1}:(9+4 \sqrt{5}-2 \sqrt{17}) v+(-9+4 \sqrt{5}+2 \sqrt{17}) w+12 \sqrt{5} u=0 \\
& K_{2}:(9+4 \sqrt{5}+2 \sqrt{17}) v+(-9+4 \sqrt{5}-2 \sqrt{17}) w+12 \sqrt{5} u=0
\end{aligned}
$$




$$
\begin{aligned}
& K_{3}:(-5-2 \sqrt{3}+4 \sqrt{5}) v+(5+2 \sqrt{3}+4 \sqrt{5}) w+12 \sqrt{5} u=0, \\
& K_{4}:(-5+2 \sqrt{5}+4 \sqrt{5}) v+(5-2 \sqrt{3}+4 \sqrt{5}) w+12 \sqrt{5} u=0,
\end{aligned}
$$

and these line pass through the point $(u, v, w)=(1,-3 / 2,-3 / 2)$. This proves (2e).

Similarly, to prove (2d) for $E_{2}$, we determine the line $N_{1}$ passing through $-Q_{1},-Q_{2}$, the line $N_{3}$ passing through $-Q_{3},-Q_{4}$. These lines are given by

$$
\begin{aligned}
& N_{1}:(-25+7 \sqrt{5}+10 \sqrt{17}+\sqrt{85}) v+(-35+13 \sqrt{5}+10 \sqrt{17}-\sqrt{85}) w+(-60+20 \sqrt{5}+20 \sqrt{17}) u=0, \\
& N_{3}:(-145+30 \sqrt{3}+63 \sqrt{5}-11 \sqrt{15}) v+(-35+30 \sqrt{3}-3 \sqrt{5}+11 \sqrt{15}) w+(-180+60 \sqrt{3}+60 \sqrt{5}) u,
\end{aligned}
$$

and the two lines pass through the point $(u, v, w)=(1,-1,-1)$. We also determine the line $N_{2}$ passing through $R_{1}, R_{2}$, the line $N_{4}$ passing through $R_{3}, R_{4}$ which are given by

$$
\begin{gathered}
N_{2}:(175+142 \sqrt{5}+10 \sqrt{17}+11 \sqrt{85}) v+(65-122 \sqrt{5}+10 \sqrt{17}-11 \sqrt{85}) w+(240+20 \sqrt{5}+20 \sqrt{17}) u=0, \\
N_{4}:(-35+10 \sqrt{3}+16 \sqrt{5}-3 \sqrt{15}) v+(-5+10 \sqrt{3}+4 \sqrt{5}+3 \sqrt{15}) w+(-40+20 \sqrt{3}+20 \sqrt{5}) u=0,
\end{gathered}
$$

and the two lines pass through the point $(u, v, w)=(1,-1,-1)$. This proves $(2 \mathrm{~d})$.

Finally, we prove that the kernel vector function of the linear matrix pencil $M(t, x, y)=$ $t I_{4}+x H_{2}+y B$ in Theorem 4 can be expressed in terms of theta functions used in the Helton-Vinnikov theorem.

Theorem 5. Using the notation and terminology of Theorem 4 , the kernel vector function $\xi=\left(\xi_{1}, \xi_{2}, \xi_{3}, \xi_{4}\right)^{T}$ expressed as a vector function on the normalized torus is given by

$$
\begin{aligned}
& \xi_{1}(v)=C_{1} \theta_{1}\left(v-Q_{2}^{\prime \prime}\right) \theta_{1}\left(v-Q_{3}^{\prime \prime}\right) \theta_{1}\left(v-Q_{4}^{\prime \prime}\right) \theta_{1}\left(v-R_{1}^{\prime \prime}\right), \\
& \xi_{2}(v)=C_{2} \theta_{1}\left(v-Q_{1}^{\prime \prime}\right) \theta_{1}\left(v-Q_{3}^{\prime \prime}\right) \theta_{1}\left(v-Q_{4}^{\prime \prime}\right) \theta_{1}\left(v-R_{2}^{\prime \prime}\right), \\
& \xi_{3}(v)=C_{3} \theta_{1}\left(v-Q_{1}^{\prime \prime}\right) \theta_{1}\left(v-Q_{2}^{\prime \prime}\right) \theta_{1}\left(v-Q_{4}^{\prime \prime}\right) \theta_{1}\left(v-R_{3}^{\prime \prime}\right), \\
& \xi_{4}(v)=C_{4} \theta_{1}\left(v-Q_{1}^{\prime \prime}\right) \theta_{1}\left(v-Q_{2}^{\prime \prime}\right) \theta_{1}\left(v-Q_{3}^{\prime \prime}\right) \theta_{1}\left(v-R_{4}^{\prime \prime}\right),
\end{aligned}
$$

for some constants $C_{j} \neq 0, j=1,2,3,4$, where $Q_{j}^{\prime \prime}$ and $R_{j}^{\prime \prime}$ are points in the normalized Abel-Jacobi variety corresponding to the point $Q_{j}^{\prime}$ and $R_{j}^{\prime}$ on the cubic curve for $j=1,2,3,4$.

Proof. Computing the numerical coordinates of the points $Q_{j}^{\prime}$, we find that the points $Q_{1}^{\prime}$ and $Q_{4}^{\prime}$ lie on the pseudo line of the cubic curve, and the points $Q_{2}^{\prime}$ and $Q_{3}^{\prime}$ lie on the oval. Changing the variables

$$
Y=\frac{1}{2}(v-w), T=-\frac{66020}{60489} u-\frac{34660}{60489}(v+w), X=\frac{467200}{20163} u+\frac{128000}{20163}(v+w),
$$

the cubic curve $F_{3}(u, v, e)=0$ is expressed in the Weierstrass canonical form

$$
-X Y^{2}+4 T^{3}-g_{2} T X^{2}-g_{3} X^{3}=0
$$

where

$$
g_{2}=\frac{7357}{76800}, g_{3}=-\frac{11023}{22118400} .
$$

The numerical values of $Q_{j}^{\prime \prime}$ in the normalized Abel-Jacobi variety corresponding to the point $Q_{j}^{\prime}$ on the cubic curve for $j=1,2,3,4$ are estimated by

$$
\begin{aligned}
& Q_{1}^{\prime \prime} \sim 0.080413336 \in[0,1], Q_{2}^{\prime \prime} \sim 0.419586665+\tau / 2 \in[0,1]+\tau / 2, \\
& Q_{3}^{\prime \prime} \sim 0.769336810+\tau / 2 \in[0,1]+\tau / 2, Q_{4}^{\prime \prime} \sim 0.730663190 \in[0,1] .
\end{aligned}
$$


By a result in [16] and the zero points relations in (2f)-(2i), the functions $\xi_{j} / \xi_{k}, 1 \leq j \neq k \leq$ 4, satisfy

$$
\frac{\xi_{j}}{\xi_{k}}(v)=C_{j k} \frac{\theta_{1}\left(v-R_{j}^{\prime \prime}\right) \theta_{1}\left(v-Q_{k}^{\prime \prime}\right)}{\theta_{1}\left(v-Q_{j}^{\prime \prime}\right) \theta_{1}\left(v-R_{k}^{\prime \prime}\right)}
$$

for some constant $C_{j k} \neq 0$, where $v$ is any point on the normalized Abel-Jacobi variety. As a consequence, the equation of the kernel vector function $\xi=\left(\xi_{1}, \xi_{2}, \xi_{3}, \xi_{4}\right)^{T}$ is derived.

In summary, the results of Theorems 4 and 5 prove that the Conjecture 1 is true for an irreducible elliptic quartic curve.

Author Contributions: Conceptualization, M.-T., C. and H.N.; formal analysis, M.-T., C. and H.N.; investigation and writing, M.-T., C.; methodology, H.N..

Funding: M.-T.C. is partially supported by the Ministry of Science and Technology, Taiwan, under MOST 109-2115-M-031-004.

Conflicts of Interest: The authors declare no conflict of interest.

\section{References}

1. Toeplitz, O. Das algebraische Analogon zu einem Satze von Fejér. Math. Z. 1918, 2, 187-197. [CrossRef]

2. Bebiano, N.; da Providência; Nata, J.A.; da Providência, J.P. Revisiting the inverse field of values problems. Electron. Trans. Numer. Anal. 2014, 42, 1-12.

3. Carden, R.L. A simple algorithm for the inverse field of values problem. Inverse Probl. 2009, 25, 115019. [CrossRef]

4. Chorianopoulos, C.; Psarrakos, P.; Uhlig F. A method for the inverse numerical range problem. Electron. J. Linear Algebra 2010, 20, 198-206. [CrossRef]

5. Kippenhahn, R. Über den wertevorrat einer Matrix. Math. Nachrichten 1951, 6, 193-228. [CrossRef]

6. Lax, P.D. Differential equations, difference equations and matrix theory. Commun. Pure Appl. Math. 1958, 11, 175-194. [CrossRef]

7. Fiedler, M. Pensils of real symmetric matrices and real algebraic curves. Linear Algebra Appl. 1990, 141, 53-60. [CrossRef]

8. Plaumann, D.; Vinzant, C. Determinantal representations of hyperbolic plane curves: An elementary approach. J. Symb. Comput. 2013, 57, 48-60. [CrossRef]

9. Helton, J.W.; Vinnikov, V. Linear matrix inequality representations of sets. Commun. Pure Appl. Math. 2007, 60, 654-674. [CrossRef]

10. Plaumann, D.; Sturmfels, B.; Vinzant, C. Computing linear matrix representations of Helton-Vinnikov curves. Operator Theory: Advances and Applications; 222; Birkhäuser: Basel, Switzerland, 2012; pp. 259-277.

11. Chien, M.T.; Nakazato, H. Computing the determinantal representations of hyperbolic forms. Czechoslov. Math. J. 2016, 66, 632-651. [CrossRef]

12. Chien, M.T.; Nakazato, H.; Yeh L. Inverse numerical range and determinantal representation. Linear Algebra Appl. 2018, 558, 79-100. [CrossRef]

13. Walker, R. Algebraic Curves; Princeton University Press: Princeton, NJ, USA, 1950.

14. Chien, M.T.; Nakazato, H. Singular points of the ternary polynomials associated with 4 by 4 matrices. Electron. J. Linear Algebra 2012, 23, 755-769. [CrossRef]

15. Fladt, K. Das Keplersche Ei. Elem. Math. 1962, 17, 73-78.

16. Hurwitz, A.; Courant, R. Vorlesungen über allgemeine Funktionentheorie und elliptische Funktionen, Grundlehren der mathematischen Wissenchaften; Band 3; Springer: Berlin/Heidelberg, Germany, 1964.

Publisher's Note: MDPI stays neutral with regard to jurisdictional claims in published maps and institutional affiliations.

(C) 2020 by the authors. Licensee MDPI, Basel, Switzerland. This article is an open access article distributed under the terms and conditions of the Creative Commons Attribution (CC BY) license (http:// creativecommons.org/licenses/by/4.0/). 